\title{
Diseño e implementación de un sistema de control de bobinas Helmholtz para la caracterización de micro y nanopartículas
}

\section{Design and implementation of a Helmholtz coil control system for the characterization of micro and nanoparticles}

RODRIGUEZ-RIZO, Reyna Michelle ${ }^{1} \dagger$, GUERRERO-SERRANO, Azdrubal Lobo ${ }^{2}$, GARCÍAGALLEGOS, Jesús Hazael ${ }^{3}$, CABAL-VELARDE, Javier Gustavo ${ }^{1 *}$

\author{
${ }^{1}$ Instituto Tecnológico Superior de Irapuato, Carretera Irapuato-Silao km 12.5 C.P. 36821, Irapuato, Gto., México. \\ ${ }^{2}$ Universidad Autónoma del Estado de Hidalgo, Área Académica de Ciencias de la Tierra y Materiales. Carr. Pachuca- \\ Tulancingo Km. 4.5, Col. Campo de Tiro, 42039 Pachuca de Soto, México. \\ ${ }^{3}$ Universidad Tecnológica de San Juan del Río Querétaro, División de Energías Renovables, Av. La Palma No. 125, Vista \\ Hermosa, 76800, San Juan del Río, Querétaro, México
}

ID $1^{\mathrm{er}}$ Author: Reyna Michelle, Rodriguez-Rizo / ORC ID: 0000-0002-8246-628X, CVU CONACYT ID: 1016313

ID $1^{\text {er }}$ Coautor: Azdrubal Lobo, Guerrero-Serrano / ORC ID: 0000-0001-5816-847X, CVU CONACYT ID: 171617

ID $2^{\text {do }}$ Coautor: Jesús Hazael, Garcia-Gallegoz / ORC ID: 0000-0002-9909-8882, CVU CONACYT ID: 175436

ID $3^{\text {er }}$ Coautor: Javier Gustavo, Cabal-Velarde / ORC ID: 0000-0002-5516-3849, CVU CONACYT ID: 99159

DOI: $10.35429 / J I D .2019 .6 .3 .19 .26$

Recibido 09 Enero, 2019; Aceptado 30 Marzo, 2019

\begin{abstract}
Resumen
En este trabajo desarrollamos un sistema de arreglos de dos pares de bobinas Helmholtz posicionadas en el eje " $\mathrm{x}$ " y "y" para la caracterización cinética de microbots en un medio líquido a diferentes densidades. Estos microbots esféricos son sintetizados con micropartículas magnéticas de magnetita embebidas en una matriz polimérica de silicón. Una de las contribuciones más específica es el desarrollo de un sistema automatizado de arreglo de bobinas Helmholtz, que, al ser desplazadas por medio de motores a pasos, el sistema de bobinas Helmholtz pasa a ser sistema de bobinas Maxwell ya que se modifican distancias, provocando un gradiente de campo uniforme en lugar de un campo magnético uniforme. Este sistema puede ser empleado en el redireccionamiento de microbots para eliminación de células cancerígenas por medio de la técnica de hipertermia; además, puede transportar medicamentos a zonas específicas para su tratamiento ya que estos microbots son biocompatibles con el cuerpo humano.
\end{abstract}

Microbots, Bobinas Helmholtz, Bobinas Maxwell

\begin{abstract}
In this work we develop a system of arrangements of two pairs of Helmholtz coils positioned on the " $x$ " and " $y$ " axis for the kinetic characterization of microbots in a liquid medium at different densities. These spherical microbots are synthesized with magnetite magnetic microparticles embedded in a polymeric silicone matrix. One of the most specific contributions is the development of an automated Helmholtz coil arrangement system, which, when moved by stepper motors, the Helmholtz coil system becomes Maxwell coil system as distances are modified, causing distances a uniform field gradient instead of a uniform magnetic field. This system can be used in the redirection of microbots for the elimination of cancer cells by means of the hyperthermia technique; In addition, you can transport medications to specific areas for treatment because these microbots are biocompatible with the human body.
\end{abstract}

Microbots, Helmholtz coils, Maxwell coils

Citación: RODRIGUEZ-RIZO, Reyna Michelle, GUERRERO-SERRANO, Azdrubal Lobo, GARCÍA-GALLEGOS, Jesús Hazael, CABAL-VELARDE, Javier Gustavo. Diseño e implementación de un sistema de control de bobinas Helmholtz para la caracterización de micro y nanopartículas. Revista del Diseño Innovativo. 2019. 3-6: 19-26

\footnotetext{
* Correspondencia del Autor (Correo electrónico: javelarde@itesi.edu.mx)

$\dagger$ Investigador contribuyendo como primer autor.
} 


\section{Introducción}

Las bobinas Helmholtz se caracterizan por ir acompañadas en pares, siendo equidistantes al radio y conectadas en serie, generando un campo magnético uniforme en el centro de las bobinas [I,III], provocando el movimiento de las micropartículas; para determinar la uniformidad del campo magnético es necesario realizar una caracterización de campo en las bobinas para saber si el punto en donde se posicionó el sensor Hall del Gaussímetro es realmente el centro de la bobina y conocer la variación de campo que genera o si hay algún campo magnético exterior al sistema que altere las mediciones. A continuación, en la Figura 1 se muestra la representación y conexión de las bobinas Helmholtz.

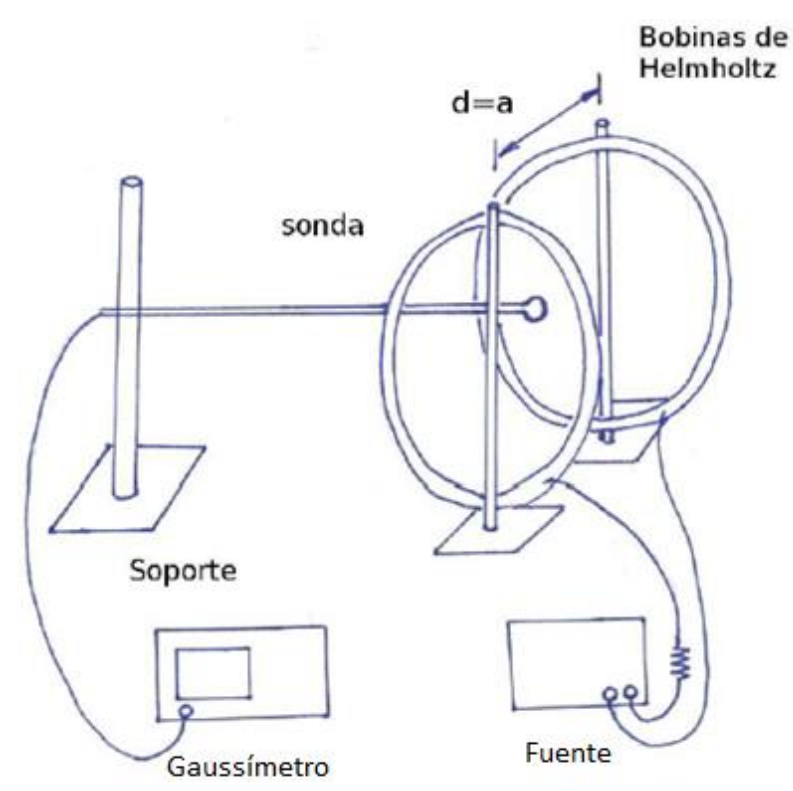

Figura 1 Representación y conexión de las bobinas Helmholtz [I]

El campo magnético generado por las bobinas Helmholtz se describe mediante la ecuación de Biot- Savart que enumera que al aplicarle una corriente a una espira de alambre este generará un campo magnético perpendicular a la corriente [IV-V].

$B_{0}=\frac{\mu_{0} N I}{r}\left(\frac{4}{5}\right)^{3 / 2}$

Donde:

$B_{0}=$ Campo magnético

$\mu_{0}=$ Permeabilidad en el vacío

$N=$ Número de vueltas propuesto

$r=$ Radio
Existen varias formas de alterar una medición para realizar la caracterización de las bobinas Helmholtz, es importante tomarlas en cuenta al momento de realizar las mediciones para no alterar el resultado esperado; una de ellas la enumera un autor que realizó una caracterización con un sistema de bobinas de Helmholtz que poseía un electroimán [II], este electroimán provocaba que las mediciones no fueran estables aún y que el sensor Hall se colocara al centro de la bobina, ya que al inyectarles corriente y generar campo magnético, este electroimán también generaba un campo magnético denominado "remanente" y provocaba inestabilidad en la lectura del campo.

Para resolver este problema el autor optó por un ciclo iterativo de corriente (ciclo de histéresis) que constituía en aumentar gradualmente la corriente en el sistema para que el campo magnético a su vez aumente y llegar a un punto tal que el campo magnético llegue a su límite y retorne a cero, pero esto no sucede; para poder lograrlo es necesario invertir la corriente y hacer el mismo proceso en el sentido contrario; de esta manera se podrán tomar las mediciones de acuerdo a las distancias requeridas sin que el campo magnético remanente afecte al campo magnético generado por las bobinas. En la Tabla 1 se muestran los valores de campo magnético a diferentes distancias obtenidos de la medición con respecto a la corriente y en la Figura 2 se muestra la caracterización del sistema de bobinas Helmholtz.

\begin{tabular}{|c|c|}
\hline $\begin{array}{c}\text { Distancia } \\
\text { entre núcleos } \\
(\mathrm{cm})\end{array}$ & $\begin{array}{c}\text { Constante de } \\
\text { proporcionalidad } \\
\text { experimental } \\
\mathrm{B}(\mathrm{T})=\alpha \mathrm{I}(\mathrm{A})\end{array}$ \\
\hline 10,0 & 0,03 \\
\hline 8,0 & 0,04 \\
\hline 6,0 & 0,05 \\
\hline 4,0 & 0,08 \\
\hline 2,0 & 0,18 \\
\hline 1,0 & 0,30 \\
\hline
\end{tabular}

Tabla 1 Medición del campo magnético a diferentes distancias [II] 


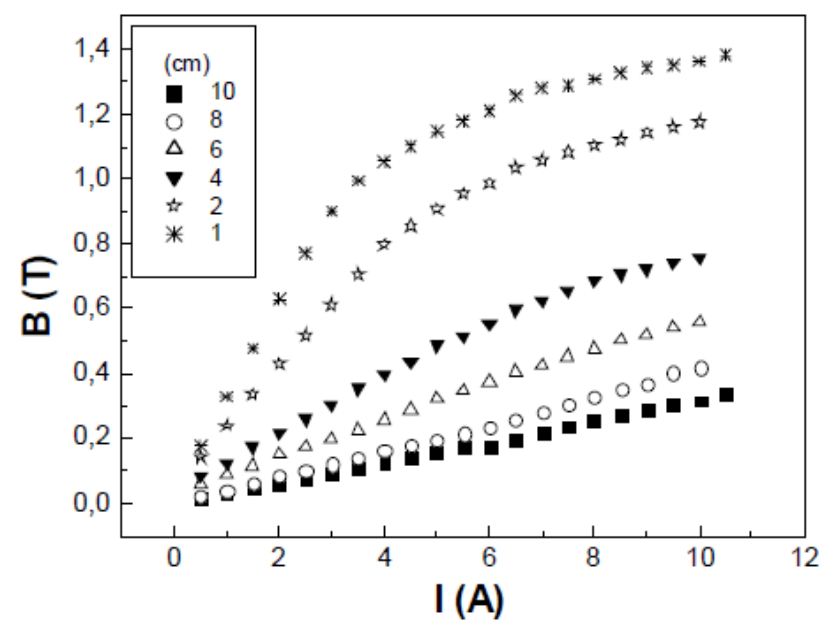

Figura 2 Caracterización de las bobinas de Helmholtz con electroimán [II]

Si el sistema no está en contacto con algún campo magnético exterior o con dispositivo que emane campo magnético, no es necesario realizar este ciclo de iteración.

Hyunchul Choi desarrolló un sistema de bobinas Helmholtz y bobinas de Maxwell para la caracterización de micropartículas o microbots [XI].

Generalmente, un microbot consiste de piezas de acondicionamiento, partes de detección, fuente de poder y herramientas de remedio. Estas partes son esenciales para aplicaciones médicas de microbots. Debido a la limitación del tamaño, sin embargo, estas partes son difíciles de integrar a un microbot, especialmente las piezas de acondicionamiento incluyen la fuente de poder. Como una solución, varios mecanismos de acondicionamiento se utilizan para impulsar el microbot desde un sitio remoto [VI].

Uno de loa muchos estudios en MEMS y la tecnología del robot se ha basado en el uso de un campo electromagnético como fuerza impulsora para microbots [VI]. Martel propone un método de actuación basado en el sistema de resonancia (MRI) [VII, VIII], la cual genera la fuerza de actuación para la locomoción de un microbot utilizando el flujo magnético uniforme y el flujo gradiente uniforme del sistema MRI. Este método puede realizar la propulsión y el seguimiento de posición del microbot mediante la repetición de propulsión de fases y fases de seguimiento en un sistema MRI [VIII].
Las bobinas de Helmholtz crean un flujo de campo magnético. Por eso, cuando un imán permanente es usado como un microbot, las bobinas de Helmholtz pueden alinear el microbot a su dirección. Las bobinas de Maxwell generan un gradiente uniforme de flujo magnético. El gradiente uniforme del flujo magnético puede producir la fuerza de propulsión para el microbot [IX].

Sitti realizó un experimento en el que se movió un robot de tamaño de micrómetro de imán permanente utilizando cinco bobinas de tipo rectángulo $[\mathrm{X}]$.

En la Figura 3 se observa el diagrama propuesto para los dos pares de bobinas, donde $\mathrm{Hx}-\mathrm{Hx}^{\prime}$ y $\mathrm{Hy}-\mathrm{Hy}^{\prime}$ son los pares de bobinas Helmholtz en el eje "x (-)" y "y (-)" respectivamente, y Mx-Mx' y My-My' son los pares de bobinas Maxwell en el eje " $x$ " $y$ " $y$ " respectivamente.

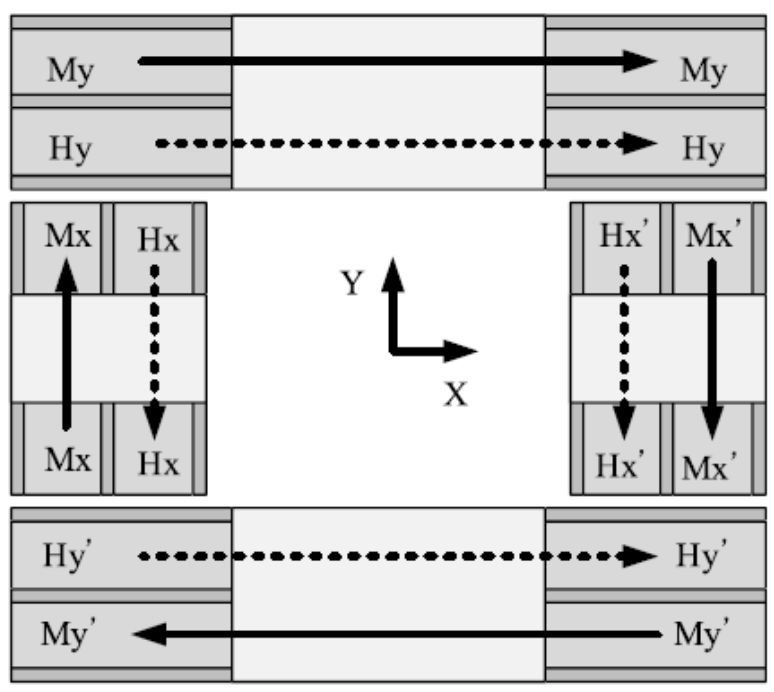

Figura 3 Diagrama propuesto para el sistema de bobinas Helmholtz y Maxwell [XI]

Aunque el sistema de bobinas tiene diferentes diámetros, aún satisface la definición principal de cada par de bobinas y cada par de bobinas de Helmholtz tiene el mismo flujo de corriente, así como cada bobina de Maxwell proporciona la misma corriente para cada par de bobinas. La magnitud y la dirección del flujo magnético generado por las bobinas de Helmholtz y las bobinas de Maxwell son gobernados por la ley de Biot-Savart, por lo tanto, el autor realizó los cálculos necesarios para el sistema de bobinas. 
La corriente aplicada a las bobinas Helmholtz (Hx, Hy) son ajustada para alinear el microbot en la dirección deseada; las bobinas de Maxwell tienen la misma corriente. El análisis numérico fue ejecutado en MATLAB, y el mapa del flujo magnético y el mapa de la fuerza de propulsión fueron simulados para la dirección deseada $\left(0^{\circ}, 45^{\circ}, 90^{\circ}, 135^{\circ}\right)$ del microbot [XI]. Las bobinas de Helmholtz propuestas pueden generar un flujo magnético en la dirección deseada. La relación de las corrientes de las bobinas de los dos pares de bobinas Helmholtz perpendiculares determina la dirección deseada del microbot. Además, para que el flujo magnético resultante de los dos pares de bobinas de Helmholtz tenga la misma magnitud, las corrientes en estas bobinas se ajustan de -2.8 a $2.8 \mathrm{~A}[\mathrm{XI}]$.

Como se muestra en la Figura 4, la simulación numérica confirma que el sistema de accionamiento electromagnético puede generar flujos magnéticos uniformes en la dirección deseada $\left(0^{\circ}, 45^{\circ}, 90^{\circ}, 135^{\circ}\right)$ [XI].

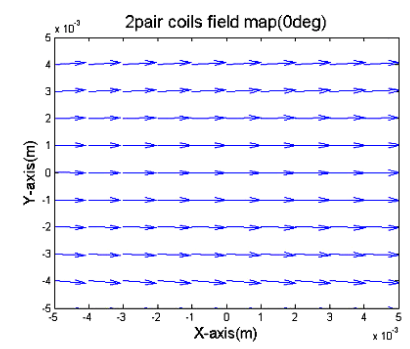

(a)

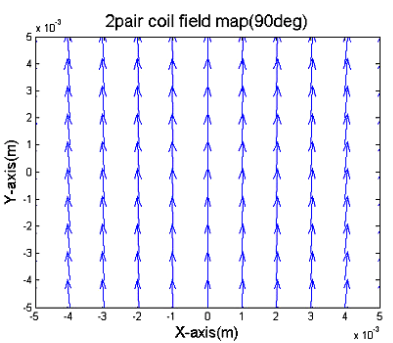

(c)

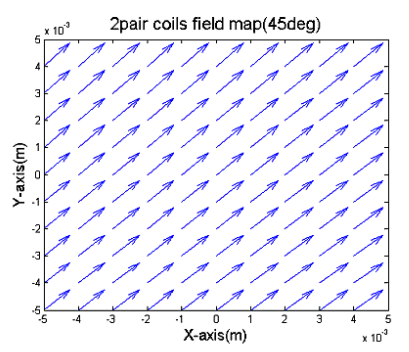

(b)

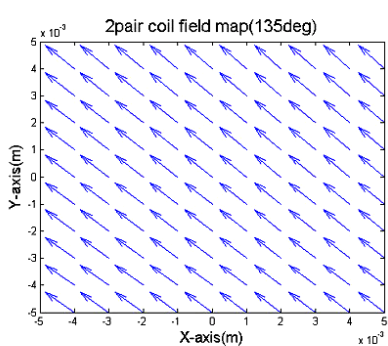

(d)
Figura 4 Mapa del campo magnético por análisis numérico. a) Dirección: $0^{\circ}$, b) Dirección: $45^{\circ}$, c) Dirección: $90^{\circ}$, d) Dirección: $135^{\circ}$ [XI]

Adicionalmente, los mapas de fuerza de propulsión también son calculados con el valor de magnetización y el gradiente de flujo magnético. La corriente de las bobinas de Maxwell tiene el mismo valor y generan una fuerza de propulsión en la dirección deseada, alineadas por las bobinas de Helmholtz. El resultado del análisis numérico se muestra en la Figura 5.
El resultado de la simulación muestra que la fuerza de propulsión uniforme es generada y los mapas de fuerza son muy similares a los patrones de los flujos magnéticos uniformes en la Figura 4. Por lo tanto, se confirmó que el microbot magnético permanente puede ser alineado y movido por el sistema de accionamiento electromagnético propuesto [XI].

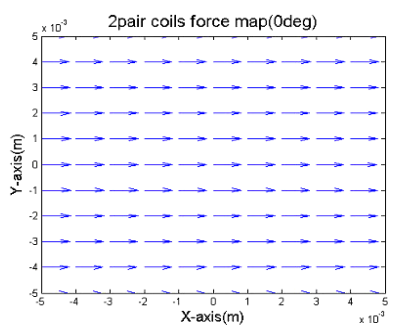

(a)

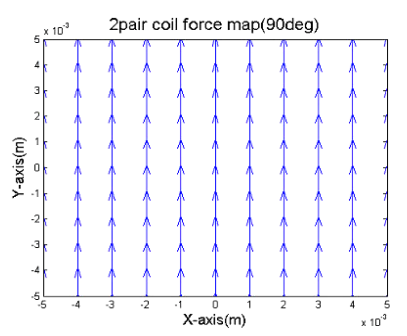

(c)

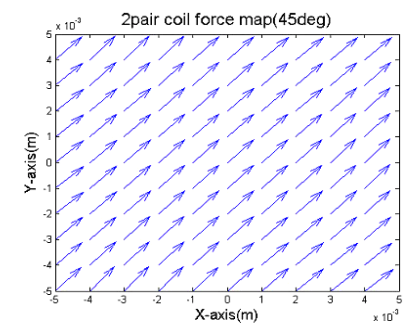

(b)

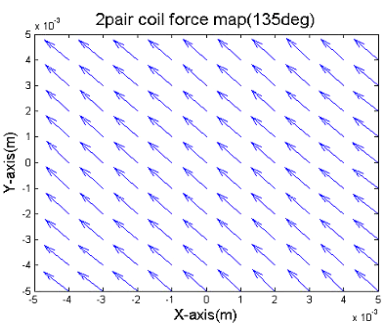

(d)
Figura 5 Mapa de fuerza por análisis numérico. a) Dirección: $0^{\circ}$, b) Dirección: $45^{\circ}$, c) Dirección: $90^{\circ}$, d) Dirección: $135^{\circ}$ [XI]

Para la evaluación del sistema de actuación electromagnética, se llevaron acabo las pruebas preliminares. En la Figura 6 se muestra la configuración experimental general para las pruebas de locomoción. El microbot fue posicionado en el centro del plato, el cual estaba lleno de aceite de silicona con alta viscosidad. El aceite de silicona genera una fuerza de arrastre a medida que aumenta la velocidad del microbot y reduce cualquier movimiento brusco del microbot. El microbot puede alcanzar una velocidad uniforme y puede ser controlado fácilmente $[\mathrm{XI}]$.

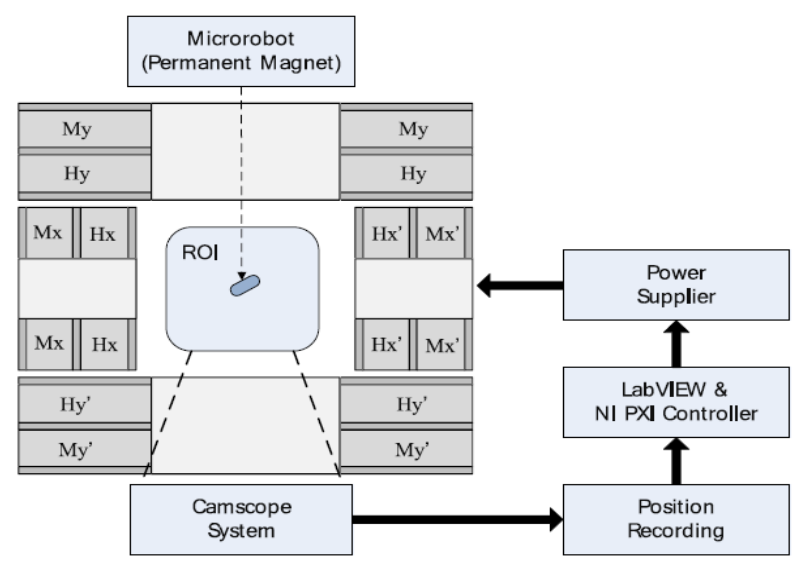

Figura 6 Configuración experimental general [XI] 
De la teoría y la simulación numérica, se confirma que la dirección del microbot es regulada por los dos pares de bobinas Helmholtz y el movimiento del microbot en esa dirección por los dos pares de bobinas Maxwell. En esta sección, a través de experimentos, se validaron los movimientos del microbot accionado por el sistema de bobina EMA propuesto. Primeramente, para el control de la corriente de las bobinas Helmholtz, la magnitud y la dirección del campo magnético fueron reguladas arbitrariamente. En la Figura 7 se muestran los resultados experimentales de la rotación del microbot [XI].
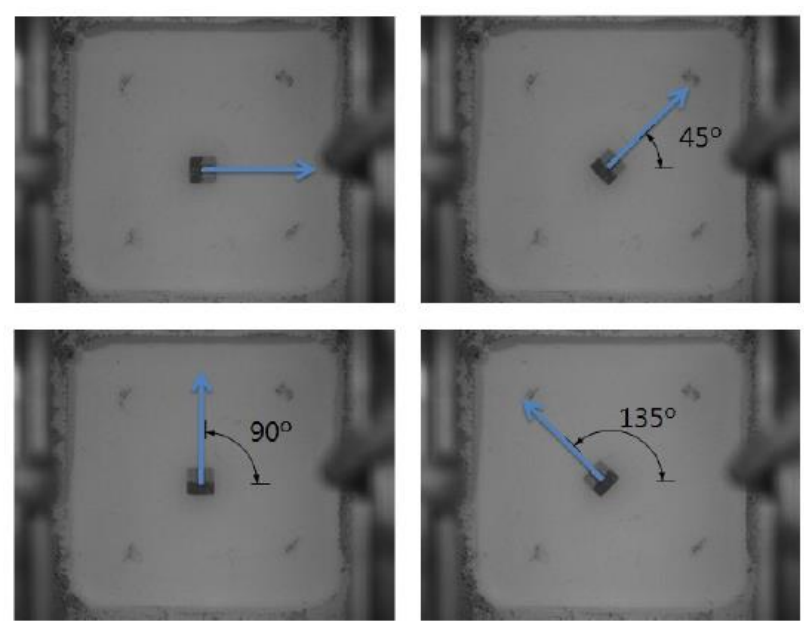

Figura 7 Rotación del microbot por las bobinas Helmholtz [XI]

En segundo lugar, el par de bobinas Maxwell proporcionó un flujo magnético de gradiente uniforme, y los dos pares de bobinas Maxwell combinados con los pares de bobinas Helmholtz se usaron para generar la fuerza de propulsión en la dirección alineada del microbot. En la Figura 8 se muestra que el microbot puede moverse en varias direcciones deseadas [XI].
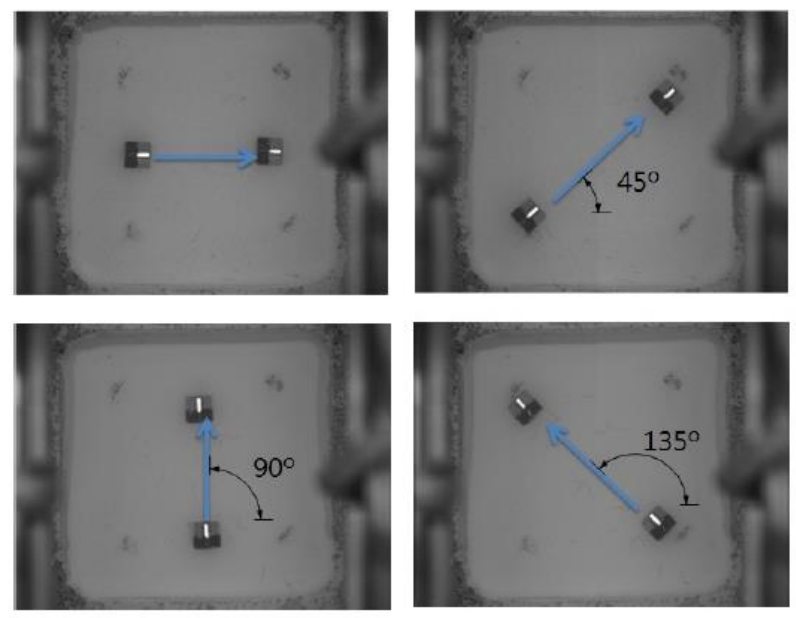

Figura 8 Propulsión del microbot en la dirección deseada usando bobinas de Helmholtz y bobinas de Maxwell [XI]

\section{Metodología}

Para el desarrollo de este proyecto fue necesario la implementación de un sistema automatizado de cuatro bobinas Helmholtz (o dos pares de bobinas) para tener mayor control sobre las esferas magnéticas poliméricas implementadas con micropartículas de magnetita embebidas en una matriz de silicón como se muestra en la Figura 9; para la creación de las bobinas se requirió implementar un diseño en FreeCAD para posteriormente hacer la impresión a 3D de los carretes de las bobinas; se hizo el alambrado de las bobinas de acuerdo con los cálculos ya establecidos dependiendo de la corriente que se le proporcionará al calibre del alambre de cobre adiamantado.

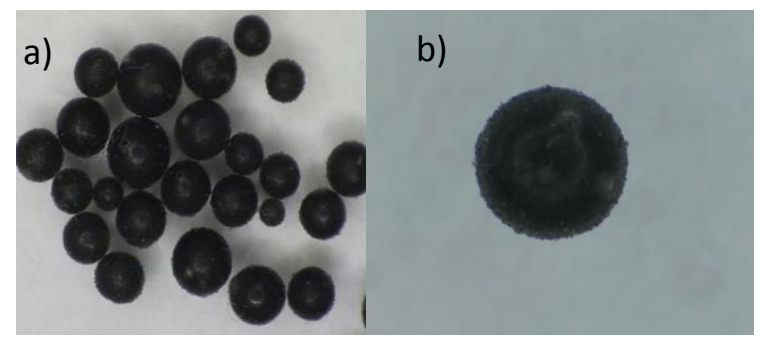

Figura 9 Microscopía óptica de esferas magnéticas de micropartículas de magnetita embebidas en una matriz de silicón (Microbot). a) Microbots a diferentes tamaños, b) Acercamiento de un microbot

Se hizo una base de aluminio estructural para colocar tres sistemas lineales (dos en el eje " $y$ " y uno en el eje " $x$ ") que se conectaran a sus respectivos motores a pasos para el desplazamiento vertical ("y") de una de las bobinas " $A$ " y para el desplazamiento horizontal (" $x$ ") para una de las bobinas "B". A continuación, en la Figura 10, se hizo un diseño en SOLIDWORKS en donde se puede observar el sistema automatizado de bobinas de Helmholtz y un sensor Hall para caracterización del sistema. Se puede observar en la imagen que el sistema de bobinas "A" posee dos sistemas de ejes lineales, esto permite mayor estabilidad al momento de desplazar la bobina, esto quiere decir, que los dos ejes lineales deben desplazarse simultáneamente para evitar errores en las mediciones. 


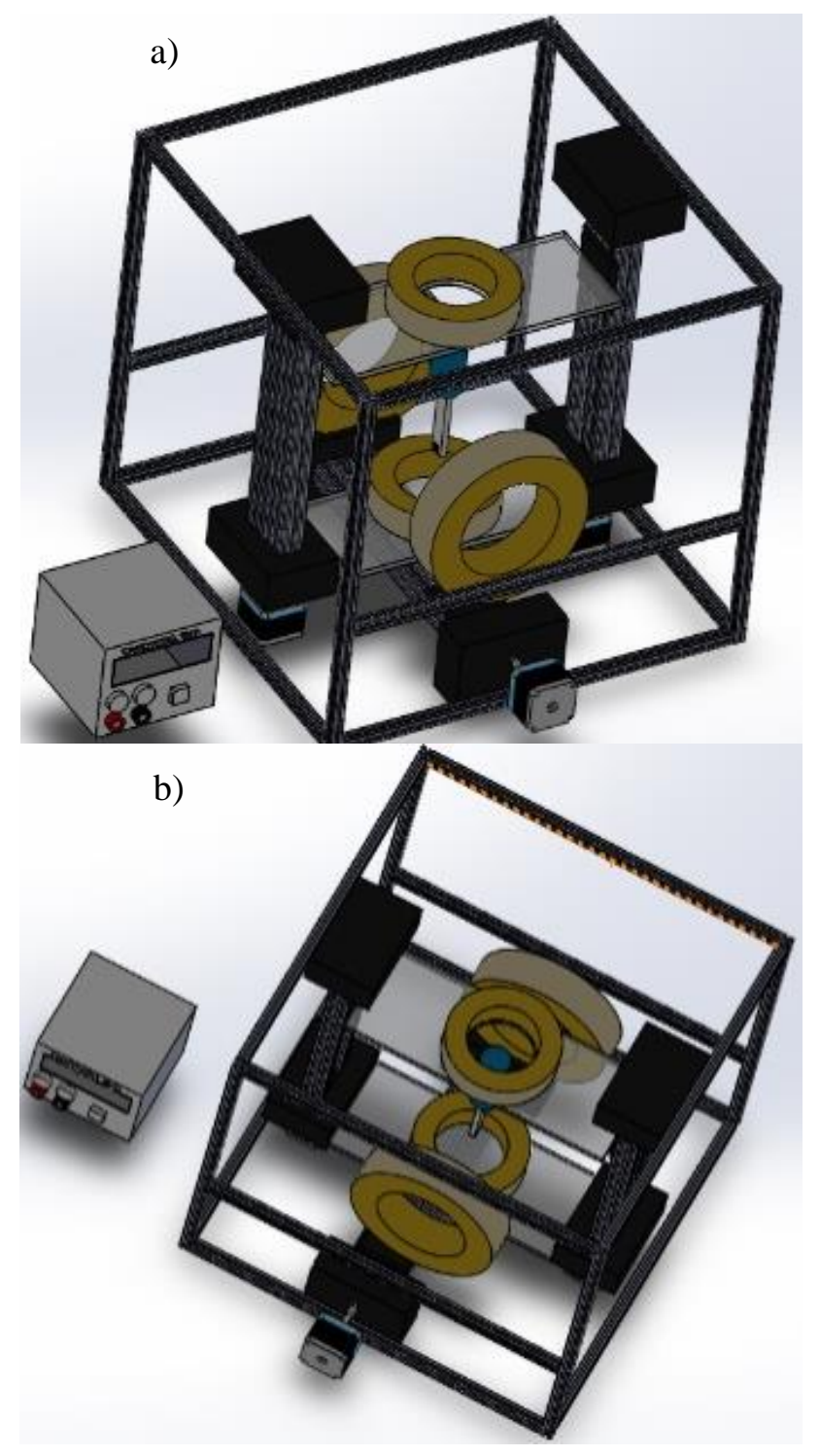

Figura 10 Sistema automatizado de bobinas Helmholtz y sensor Hall para la caracterización a) Vista lateral del sistema y b) Vista superior del sistema

Para el control de los motores a pasos se utilizó como puente de comunicación una tarjeta Arduino Uno y fue programada por medio de una interfaz gráfica creada en LabVIEW que permite el desplazamiento de los ejes lineales trabajando con distancias. En la Figura 11, se muestra un diagrama de flujo de la programación para el desplazamiento de las bobinas.

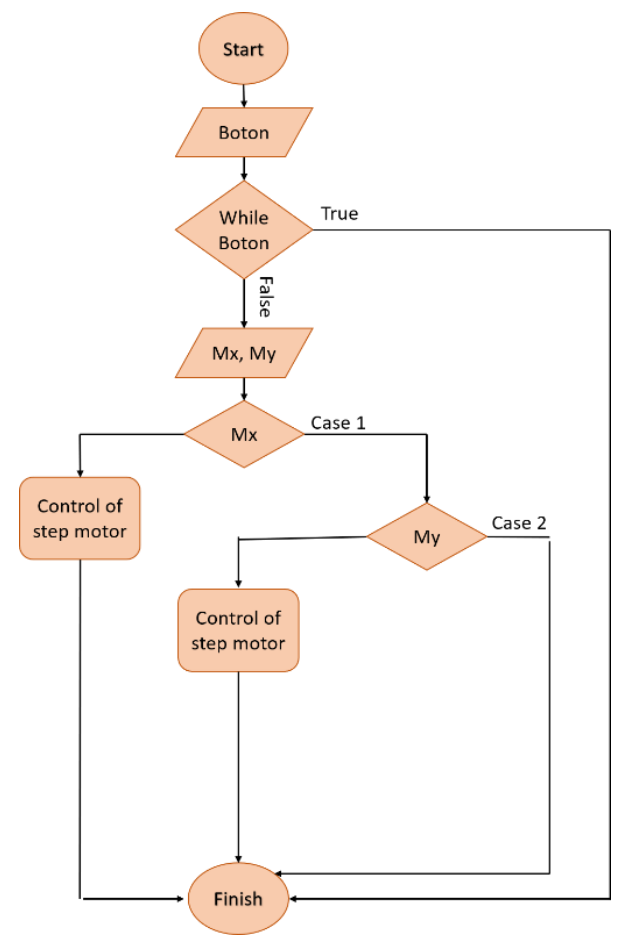

Figura 11 Diagrama de programación para el desplazamiento de los ejes lineales

\section{Resultados}

El programa realizará básicamente un algoritmo que permitirá el movimiento paso a paso de los motores a pasos, como se sabe, LabVIEW no posee una librería que controles los motores a pasos directamente; es por eso que fue necesario hacer una interfaz que controle los pasos pulso por pulso dependiendo de la estructura del motor, el motor que se utilizó gira $1.8^{\circ}$ por paso permitiéndonos hacer los cálculos necesarios para que el motor gire los pasos requeridos a la distancia deseada. Este procedimiento se realizó con los tres motores y tomando en cuenta que en el eje "y" es necesario que los motores lleven sincronía a la hora del desplazamiento.

Teniendo el sistema y el control de motores se procede a la caracterización de las bobinas "A y "B". Se pretende caracterizar de manera individual cada par te bobinas, esto quiere decir, que se caracterizará sólo las bobinas " $\mathrm{A}$ " y después las bobinas " $\mathrm{B}$ "; esto se logrará conectando en serie las bobinas e inyectándole un voltaje inicial de $1.8 \mathrm{~V}$ y una corriente determinada por la resistencia del alambre de cobre y el voltaje proporcionado, y a medida que se toman las mediciones tanto el voltaje como la corriente irán aumentando hasta 3 A en la primera medición y en la segunda medición 5 A; este proceso es para la caracterización del sistema de bobinas " $\mathrm{A}$ " tanto para el sistema de bobinas "B". 
En la Figura 12 se muestran los valores de campo magnético ya caracterizados de la bobina "A" en a) la medición 1 y b) la medición 2 y con el sensor Hall posicionado en el centro de las bobinas de manera horizontal.
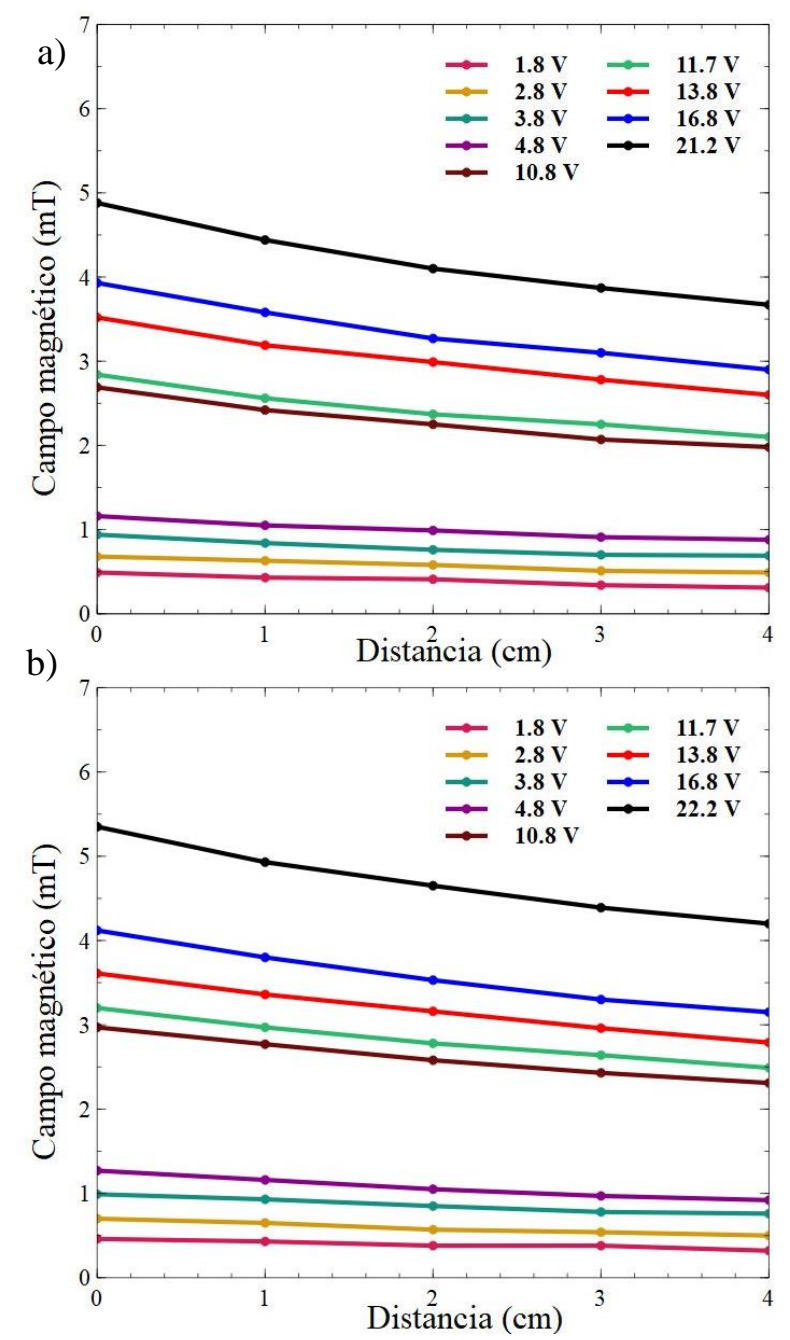

Figura 12 Caracterización de las bobinas "A" a) Medición 1 y b) Medición 2

En la Figura 13, se muestran los valores del campo magnético caracterizado de la bobina "B" en a) la medición 1 y b) la medición 2 con el sensor Hall posicionado en el centro de las bobinas de manera vertical.
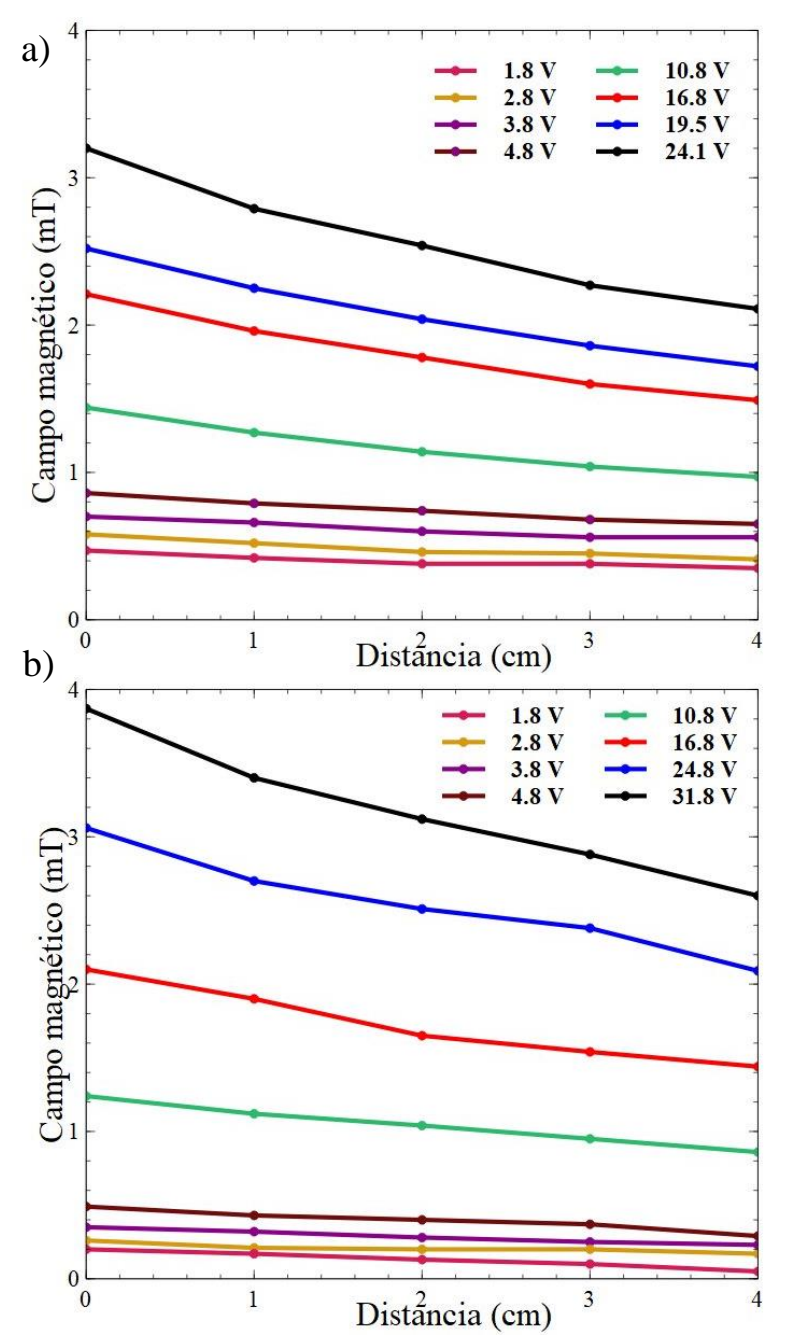

Figura 13 Caracterización de las bobinas "B" a) Medición 1 y b) Medición 2

\section{Conclusión}

Gracias al sistema automatizado de bobinas Helmholtz y el uso de una cámara, se pudo observar el desplazamiento de la micropartícula y por ende obtener la gráfica en donde se puede observar que su desplazamiento es lineal debido al campo magnético que generan las bobinas de Helmholtz. Además, la velocidad de desplazamiento de la micropartícula aumenta conforme el campo magnético aumenta, en cambio, si la distancia de las bobinas aumenta, la velocidad de dichas partículas si aumenta, pero no de la misma manera que si las distancias de las bobinas de Helmholtz permanecen en su posición original.

\section{Referencias}

Abbott, J. J., Nagy, Z., Beyeler, F., \& Nelson, B. J. (2007). Robotics in the small, part I: microbotics. IEEE Robotics \& Automation Magazine, 14(2), 92-103. 
Casañas B., Instrumentación y control de la técnica de magnetoimpedancia para materiales ferromagnéticos suaves, Universidad Nacional Autónoma de México, 37-77.

Chanu, A., \& Martel, S. (2007, August). Realtime software platform design for in-vivo navigation of a small ferromagnetic device in a swine carotid artery using a magnetic resonance imaging system. In 2007 29th Annual International Conference of the IEEE Engineering in Medicine and Biology Society (pp. 6584-6587). IEEE.

Choi, H., Choi, J., Jang, G., Park, J. O., \& Park, S. (2009). Two-dimensional actuation of a microrobot with a stationary two-pair coil system. Smart Materials and Structures, 18(5), 055007.

Fano, W. G., Alonso, R., \& Quintana, G. (2017). El Campo Magnético Generado por las Bobinas de Helmholtz y sus Aplicaciones a Calibración de Sondas. Elektron, 1(2), 91-96.

Floyd, S., Pawashe, C., \& Sitti, M. (2008, May). An untethered magnetically actuated microrobot capable of motion on arbitrary surfaces. In 2008 IEEE International Conference on Robotics and Automation (pp. 419-424). IEEE.

Hayt, W. H., Buck, J. A., \& Pedraza, C. R. C. (2006). Teoría electromagnética. McGraw-Hill. Martínez, A. C. A., \& Valdez, J. F. (2017). Diseño, Construcción Y Caracterización De Un Dispositivo Para Generar Un Campo Magnético Uniforme. JÓVENES EN LA CIENCIA, 2(1), 252-257.

Mathieu, J. B., Martel, S., Yahia, L., Soulez, G., \& Beaudoin, G. (2003, May). Preliminary studies for using magnetic resonance imaging systems as a mean of propulsion for microrobots in blood vessels and evaluation of ferromagnetic artefacts. In CCECE 2003-Canadian Conference on Electrical and Computer Engineering. Toward a Caring and Humane Technology (Cat. No. 03CH37436) (Vol. 2, pp. 835-838). IEEE.

Torres, J. I., Muñoz, B. C., \& Ramírez, W. M. (2007). Caracterización y calibración automática de bobinas de Helmholtz en DC. Scientia et technica, 1(34).
Yesin, K. B., Vollmers, K., \& Nelson, B. J. (2006). Modeling and control of untethered biomicrorobots in a fluidic environment using electromagnetic fields. The International Journal of Robotics Research, 25(5-6), 527-536. 